\title{
Barbara, femme coûte que coûte. Regard psychanalytique
}

\author{
Florent POUPART (Toulouse)
}

\section{Summary}

Psychoanalysis has always needed myths and literature to explain the mysteries of human psychology. This is particularly true for the feminine part of our personality. By means of autobiographical statements and song texts by Barbara, the author shows how this French singer and poet has succeeded in putting images and words on the feminine: receptiveness, concavity, affectivity, sensibility, sensuality, etc. But Barbara's work also testifies to a subversion of the feminine following a traumatic sexual experience, which then could turn into a form of melancholy.

Que signifie être femme? Les études de genre (Gender Studies) interrogent depuis un demisiècle les rapports entre les sexes et les facteurs sociaux qui déterminent les identités de genre. Pour la psychanalyse, en revanche, le féminin n'est pas un genre : il est une position subjective, qui constitue l'une des facettes du fonctionnement psychique de chacun, avec laquelle doivent composer, aussi bien les hommes que les femmes (bien que de façon différente) : c'est la théorie d'une bisexualité psychique universelle, soutenue par Sigmund Freud dès les années 1890. Si la psychanalyse a pu s'emparer du concept de genre, c'est toutefois en maintenant la distinction entre identité de genre (se sentir homme ou femme) et position identificatoire au sein de cette bisexualité psychique postulée, qui relève d'une mise en tension d'aspects masculins et féminins au sein de la vie psychique, par identification du sujet aux deux figures féminine-maternelle et masculine-paternelle mises en scène dans la fantasmatisation œdipienne, articulée autour de la scène primitive, qu'on se situe biologiquement et identitairement comme homme ou comme femme ; c'est, également, en refusant l'a priori théorique d'une détermination exclusivement sociale, au détriment de tout facteur biologique, dans la construction de l'identité sexuée. (Cf. Korff-Sausse 2010)

Comment, dès lors, définir le féminin dans une conception psychanalytique qui fait de la sexualité le centre de gravité de la construction identitaire et le moteur de la vie psychique? S’il est justifié de postuler une influence biologique sur la différence des sexes, c'est d'abord dans la différence anatomique entre ceux-ci qu'elle s'enracine (Cf. Freud 2004, 123). Par analogie, alors que le masculin s'exhibe et se déploie dans une activité bruyante et manifeste, 
le féminin serait caché (à l'instar de son sexe), et finalement indicible. Il est toutefois sujet à d'infinis débats en psychanalyse : d'une part, les tenants d'un féminin ‘ phallocentré ', qui considèrent la femme comme un garçon manquant, castré, qui n'est attirée sexuellement vers l'homme qu'en vertu de ‘ l'envie du pénis )(elle veut jouir du sexe de l'homme en soi, à défaut d'en avoir un à soi); d'autre part, ceux qui soutiennent l'existence d'une essence féminine à part entière, irréductible à la passivité, à l'incomplétude, au manque, à la castration, à la perte, bref, à un négatif du masculin. C'est le cas d'une large part des auteurs psychanalytiques contemporains, notamment au sein de l'École française : Jacqueline Schaeffer, Catherine Chabert, Jacques André, Monique Schneider qui, suivant les voies ouvertes par Karl Abraham, Ernest Jones, Karen Horney, Melanie Klein, entre autres, poursuivent l'élaboration d'une compréhension psychanalytique d'un féminin autonome et positif : ni en miroir du masculin, ni dans son ombre, ni enfin confondu avec lui. Mais dès lors, comment le définir?

Lorsqu'il s'agit de situer l'essence du féminin autrement qu'en référence à la passivité, Freud lui-même avoue son impuissance : "Si l'on tente de les ramener à des principes plus originaires, la masculinité se volatilise en activité, et la féminité en passivité, ce qui est trop peu. » (Freud 1974, 70) Il n'a pas varié sur ce point : le couple masculin/féminin serait l'ultime degré d'élaboration d'un autre, réputé plus élémentaire, le couple activité/passivité. Où se situe alors l'écart entre la passivité et le féminin ? Pour tenter de répondre, on peut considérer la dimension foncièrement paradoxale de la position identificatoire féminine, toute entière condensée dans le verbe réfléchi « s'abandonner ( (cf. Poupart 2016, 711) : on pense ici à la danseuse qui s'abandonne au " guidage " du danseur, comme l'hypnotisé aux suggestions de l'hypnotiseur, l'amoureux à l'être passionnément aimé, ou encore la foule au leader charismatique (cf. Freud 1973, 134-141). Le féminin ' génitalisé > consisterait alors à accompagner le partenaire, à se fondre dans le mouvement qu'il suggère sans l'imposer, et d'une façon plus générale à accueillir l'autre en soi, métonymiquement représenté par son désir (dans les suggestions), et éventuellement par son pénis (dans la pénétration sexuelle).

Pour définir les contours de cette part féminine de la subjectivité humaine, il a fallu à la psychanalyse se tourner vers les hystériques, les mystiques, les mythes, la littérature. Nous inscrivant dans cette tradition, nous proposons ici de repérer ce qui, dans l'œuvre écrite de Barbara (qu'il s'agisse des chansons dont elle est l'auteure ou de son autobiographie) relèveraient d'une poésie du féminin dont l'immense mérite est de figurer l'invisible, de nommer l'indicible.

\section{La réceptivité dans les textes de Barbara}

Dans Les mots de Barbara, Joël July (2004) défend, envers et contre la chanteuse elle-même, la dimension poétique de son ouvre. Relativement au féminin, nous nous attarderons sur deux aspects de ses textes qui traduisent la réceptivité : la concavité et la sensorialité. July $(2004,280)$ insiste sur l'usage récurrent chez Barbara des creux du corps à connotation 
hautement érotique (au creux du cou, de la main, des reins, du bras), mais aussi, plus métaphoriquement, le creux du jour, du lit, de moi, ou encore de l'hiver (lorsque le corps devient trop fatigué et douloureux pour accueillir le corps, le désir et l'amour d'un homme). Ailleurs, c'est aussi le creux de l'autre qu'elle évoque : il prend alors une connotation plus contenante/maternelle pour l'accueillir elle. Ainsi, elle se cale volontiers au creux du bras d'un homme comme, dans l'enfance, elle se consolait auprès de sa grand-mère Granny en se réfugiant au creux de son épaule.

Les récits érotiques chez Barbara figurent un homme qui s'approche, et son corps à elle qui s’y abandonne. Le verbe < plier > vient souvent figurer l'abandon : "Quand il a touché mon cou nu, j'aurais aimé coucher mes reins, j’aurais aimé plier mon cou » ( Lily passion ", 198611). Dans "À peine " (1970), la chanson " la plus sensuelle de Barbara " (July 2004, 277), elle raconte une journée d'amour de deux amants qui ne quittent pas la chambre de l'aube à la nuit : « je suis la vague où tu te noies ». À nouveau, la référence aux reins figure le caractère cavitaire du corps propre, auquel répond, en miroir, la convexité des mains de l'amant, prolongement pénétrant du sien : «déjà ta main s'est glissée, légère, légère [...] tu frôles mes reins".

Le verbe < venir > et ses dérivés sont omniprésents dans les textes de Barbara, comme métaphore de la position active-masculine qui la place, elle, en position féminine-concave : "Dis, quand reviendras-tu ?" (dans la chanson du même nom, Barbara 1964a), ou encore " Mon amour, il faut que tu viennes!» (Barbara 1972a) comme appel puissant adressé à l'autre pénétrant. D'autant que < venir > résonne aussi comme écho de l'orgasme (masculin et féminin), ( petite mort > à laquelle chacun des partenaires du couple génital s'abandonne, seul et passif, ne fût-ce qu'un instant (cf. André 2007, 122).

La sensorialité apparait dans les mots de Barbara comme l'autre pôle privilégié d'accès au féminin. Dans ses chansons comme dans son autobiographie, l'accent est toujours mis sur la sensation et le ressenti plutôt que sur la pensée et l'analyse. July écrit que «Barbara prend plaisir à montrer surtout l'instant du contact, l'instant où la peau sensible du cou va recevoir l'épiderme qui fera frémir et plus particulièrement ‘ plier > " (2004, 277). Mais avant même les images et les mots du texte, c'est d'abord un rapport sensuel à la sensorialité du chant luimême dont elle témoigne. C'est ainsi que les mots chantés sont vécus et décrits par Barbara comme des corps étrangers pénétrants $(1998,148)$ :

On ne sait pas d'où viennent les mots ; quand tu chantes, ils se mâchent, s'allongent, se distordent, se consument, déboulent de ta gorge à tes lèvres, redescendent dans ton corps, dans le pli de ta taille, dans ta hanche ; ils t'obligent à tendre la jambe, à plier l'épaule, à courber l'échine, à redresser les reins le long desquels ils se faufilent jusqu’à redescendre jusqu'aux extrémités où ils irradient parfois comme une douleur ou un plaisir intenses. 
Un tel rapport aux mots s'étend aussi à l'acte créateur de l'auteure $(1998,149)$ :

Nous sommes en 1962. «À Rémusat , je ressens le désir d'écrire ; le besoin d'écrire. Jusqu’à présent, je n’ai écrit que deux chansons, mais je sens qu'en moi les mots bougent et cognent. Ils veulent sortir, les mots ; ils s'agitent, s'entremêlent, se conjuguent pour dire ce que je n’arrive pas encore à expliquer. Ils vont filtrer, sourdre, jaillir de mes veines. Ils me font peur et me fascinent à la fois. Je ne comprends pas d'où ils viennent, ni l'état de fébrilité où je suis lorsqu'ils me viennent.

Les mots, chez Barbara, ne sont pas des fétiches, des possessions précieuses à garder secrètes, à livrer au compte-goutte, à monnayer, à utiliser pour impressionner ou sidérer, à exhiber fièrement comme un trophée ; les mots ne lui appartiennent pas, ils sont des corps étrangers qui la parcourent, l'excitent, qu'elle ne maîtrise pas, qu'elle ne peut qu'accueillir et accompagner dans une tension voluptueuse lorsqu'ils la traversent car ce sont eux qui, tel l'amant désiré, viennent à elle. Ainsi évoque-t-elle aussi les mots qu'elle a lus, et qui lui échappent par une régression/conversion vers le corps $(1998,151)$ :

[...] les mots, au lieu de rester dans ma mémoire visuelle, se sont agglutinés dans ma mémoire tactile, et, aujourd'hui, je sais que ce sont ces mots-là qui bougent au bout de mes doigts, qui cherchent à sortir du bout de mes doigts, de tout mon corps. En chantant, je retrouve cette sensation de mots jadis avalés, déglutis, engloutis, qui remontent douloureusement par ma gorge avant que je ne les exhale avec violence ou douceur dans une chanson.

Cette réceptivité, souvent voluptueuse, est parfois au contraire vécue dans la douleur et l'angoisse, comme lorsque ces corps étrangers internes sont décrits, dans "Lily Passion " (1986), comme des intrus, ‘ intromis > en elle par un énigmatique " on» :

Et les mots qui sortent de ma gorge, je ne les connais pas : des mots qu'on a plantés là, des mots qui me font mal et qui m'étouffent ; alors je les crie, je les vomis pour pouvoir respirer, pour vivre...

Dans ses Mémoires, elle écrit que " les mots se remettent à écrire tout seuls " $(1998,160)$; l'acte d'écrire prend chez elle une forme comparable à l'écriture automatique des spirites, ou à l'hallucination psychomotrice (automatisme mental) des psychotiques : ici comme ailleurs, le vécu d'influence apparaît comme une modalité d'expression du féminin (cf. Poupart 2017). 


\section{La confusion activité/passivité, indice d'un "féminin mélancolique "} (Chabert 2007)

Dans son autobiographie, Barbara raconte le souvenir de l'inceste paternel subi à Tarbes à l'âge de dix ans :

J'ai de plus en plus peur de mon père. Il le sent. Il le sait. J'ai tellement besoin de ma mère, mais comment faire pour lui parler ? Et que lui dire ? Que je trouve le comportement de mon père bizarre? Je me tais. Un soir, à Tarbes, mon univers bascule dans l'horreur. J'ai dix ans et demi. Les enfants se taisent parce qu'on refuse de les croire. Parce qu'on les soupçonne d'affabuler. Parce qu'ils ont honte et qu'ils se sentent coupables. Parce qu'ils ont peur. Parce qu'ils croient qu'ils sont les seuls au monde avec leur terrible secret. (Barbara 1998, 31)

Au moins trois de ses chansons font explicitement ou implicitement référence à son père : Joël July parle du "triptyque de Jacques Serf " ${ }^{2}(2012,25)$. À chaque fois, la dialectique activité/passivité, dont témoigne sans cesse Barbara s'agissant de ses passions amoureuses, se brouille ou se renverse en son contraire. Dans "L'aigle noir » (1970), on observe ainsi une inversion remarquable de l'usage qu'elle fait habituellement des métaphores érotiques main/cou. Dans le reste de l'œuvre, le cou figure par excellence la concavité du corps, le creux, à l'instar des reins ; en miroir, la main de l'amant semble représenter son pénis, qui touche, frôle, cherche le corps de Barbara : " déjà ta main s'est glissée, légère, légère [...] tu

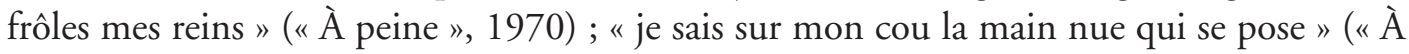
mourir pour mourir », 1964b) ; "Il n'y en a qu'un qui sait poser ses mains au creux de mon cou, au creux de mes reins" ("Ni belle ni bonne ", 1964b) ; "Ta bouche à mes cheveux et ta main sur mon cou " ("Parce que (je t'aime) », 1967) ; "Je serai obéissante quand tes mains caresseront mon cou, mes hanches, ma taille " (Je serai douce », 1970) ; " tes mains à mon cou nu, comme des algues brunes, se sont enroulées " ("Clair de nuit ", 1972b) ; "Quand il a touché mon cou nu, j’aurais aimé coucher mes reins, j’aurais aimé plié mon cou » ("Lily Passion », 1986). Dans «L'Aigle noir » (1970) en revanche, le cou de l'oiseau, de par sa forme caractéristique, devient convexe, objet pénétrant, et la main de Barbara, à la faveur d'une légère torsion, se fait creux, réceptacle : "Dans ma main, il a glissé son cou ". Si la chanson "L'Aigle noir ", l'un de ses grands succès, a pu être considérée comme un récit onirique mettant symboliquement en scène l'inceste paternel qu'elle a subi enfant, c'est notamment à cause du vers " Il m’était revenu », qui résonne étrangement avec plusieurs vers de «Nantes " (1964b), où la référence paternelle n’est pas contestable : "Il me revenait en plein cœur [...] Voilà qu'il m’était revenu [...] Il était revenu un soir ».

Dans " Nantes ", justement, Barbara fait le récit du voyage qui l'amène jusqu’à son père mort, et il est frappant de noter qu'insensiblement au fil de la chanson, on ne sait plus qui 
vient à qui. C'est d'abord elle qui "fait le voyage " jusqu’à lui, en répétant l'adresse de sa destination « 25, rue de la Grange-aux-Loups » :

Nantes m'était alors inconnue

Je n’y étais jamais venue

Il avait fallu ce message

Pour que je fasse le voyage

Puis aussitôt après, c'est le père (à la fois disparu depuis dix ans et revenant, paradoxalement, à l'occasion de sa mort), qui est décrit comme ( (re)-venant > à elle, et Barbara se retrouve assignée en position passive d'attente :

Après bien des années d'errance,

Il me revenait en plein cœur [...]

Depuis qu'il s'en était allé

Longtemps je l'avais espéré

Ce vagabond, ce disparu

Voilà qu'il m'était revenu

Puis enfin :

Il était revenu un soir

Et ce fut son dernier voyage

Ce n'est plus elle qui fait le voyage à lui, mais lui qui fait le voyage à elle.

Cette confusion des rôles, des positions active et passive, figurée dans le champ lexical des allées et venues cher à Barbara (si souvent utilisé pour figurer la relation sexuelle comme les vicissitudes conjugales : qu'on songe à "Vienne »...), se retrouve dans la chanson "Au cœur de la nuit» (1967), qui raconte un souvenir d'une nuit d'enfance, et que l'on peut entendre comme le récit de la première scène d'abus sexuel par le père. Et à nouveau, on ne sait plus qui vient à qui :

J'allais à demi éveillée

Longeant une allée obscure,

J'allais à demi éveillée

Guidée par l'étrange murmure.

Il y eut, je me le rappelle

Surgissant de l'allée obscur

Il y eut un bruissement d'ailes

Là, tout contre ma figure. 
C'était au cour de la nuit

C'était une forêt profonde. [...]

Qui es-tu pour me revenir?

Et veux-tu que vers toi je vienne?

S'il faut $j$ 'irai encore

Dans ces vers, qui condensent l'évocation d'une figure onirique déjà rencontrée dans "L'Aigle noir » (" un bruissement d'ailes ») et le thème du > re-venant > "Qui es-tu pour me revenir ?»), on ne sait plus qui va à qui, qui pénètre qui, qui séduit qui.

Cette confusion actif/passif est caractéristique de la « confusion de langue > décrite chez les victimes d'inceste. Le psychanalyste hongrois (et proche disciple de Freud) Sándor Ferenczi a repéré en 1932 le mécanisme d'identification à l'agresseur caractéristique des situations d'abus sexuel intrafamilial :

Les enfants se sentent physiquement et moralement sans défense, leur personnalité encore trop faible pour pouvoir protester, même en pensée, la force et l'autorité écrasante des adultes les rendent muets, et peuvent même leur faire perdre conscience. Mais cette peur, quand elle atteint son point culminant, les oblige à se soumettre automatiquement à la volonté de l'agresseur, à deviner le moindre de ses désirs, à obéir en s'oubliant complètement, et à s'identifier totalement à l'agresseur. (Ferenczi 1996, 130)3

Mais en s'identifiant ainsi à son agresseur, l'enfant fait sienne la culpabilité de celui-ci, et pourra mettre en œuvre une série de stratégies d'autopunition : c'est ce que Catherine Chabert (2003) a développé sous le concept de ‘ féminin mélancolique `.

\section{Les destins du ‘ féminin mélancolique > chez Barbara}

Catherine Chabert enracine ‘ le féminin mélancolique > dans les travaux freudiens sur les fantasmes originaires. Freud décrit en 1918 un scénario fantasmatique originaire (c'est-àdire à la fois à l'origine de la vie fantasmatique, phylogénétiquement transmis, donc universel, et relatif aux origines du sujet), dans le lequel le sujet est en position d'observateur d'une scène sexuelle entre ses parents : il est témoin de sa propre conception. Ce fantasme articule deux autres fantasmes originaires : la castration (la situation de coït parental met en scène deux figures dont l'une est nantie et l'autre châtrée, autour de quoi va s'organiser la différence des sexes et le complexe de castration chez l'enfant, garçon ou fille) et la séduction (l'enfant est séduit/excité par ce que les adultes offrent/soumettent à son regard).

Chabert souligne la dimension " hystérique " du fantasme de séduction : l'enfant est passivement séduit par la scène dont il est témoin, grâce à un retournement de l'activité en passivité : « ce n’est pas moi qui le désire, c'est lui qui me séduit »(Chabert 2007, 36s). Toutefois, pour Chabert, "lorsque la voie passive n'est pas accessible le retournement de 
l'activité en passivité n'est plus possible et le fantasme < hystérique > de la séduction laisse la place à une autre version ", qu'elle qualifie de " mélancolique " (Chabert 2007, 36s). Dans le féminin mélancolique, l'enfant, ne supportant pas la position passive à laquelle il est assigné par le fantasme, s'en défend par une fixation à la position active : « c'est moi qui ai séduit l'adulte ". Dès lors, il est acculé par un sentiment de culpabilité inconscient qui le pousse à l'autopunition. Chabert $(2007,39)$ ajoute que le prix à payer de la version mélancolique du fantasme de séduction est l'extinction des mouvements pulsionnels. On peut s'interroger sur les destins du féminin mélancolique chez Barbara, et émettre quelques hypothèses, à partir d'éléments de son histoire rapportés par elle.

Son autobiographie révèle d'abord une auto-destructivité importante à l'adolescence : Barbara décrit une période de marginalité, une vie d'errance entre Bruxelles et Charleroi entre 20 à 22 ans, qui l'amène à vivre dans la rue, dans des squats d'artistes, ou ponctuellement dans des hôtels qu'elle ne peut pas payer. Cela durera trois ans, et l'amènera à flirter avec la prostitution, avant de rentrer en France sans papiers grâce à l'intervention d'un proxénète qui négocie avec les douaniers, avant de lui proposer de la mettre sur le trottoir à Paris... (elle en fera une chanson : "Monsieur Victor ", 1981). On voit ici comment Barbara, à l'orée de l'âge adulte, s'est livrée à une mise en danger d'elle-même grave et répétée.

Elle fait état de nombreuses et fréquentes hospitalisations tout au long de sa vie, notamment en lien avec une douleur chronique qui l'amènera à développer une dépendance à la cortisone. Barbara évoque une douleur aux reins qui apparaît lorsque son père abandonne la famille (elle a 17 ans) : " Je me souviens de la douleur lancinante que je ressentis dans le bas des reins ; elle reviendra à chaque bouleversement intense de ma vie. " $(1998,70)$ Les reins, métaphore privilégiée par Barbara pour figurer le creux sexuel féminin, est aussi le choix d'élection d'une somatisation dans ses douleurs chroniques, notamment lorsqu'elle apprend par un appel téléphonique la mort de son père après dix ans d'absence : "Une douleur brûlante glisse dans mes reins. " $(1998,127)$ La solution somatique et l'agir autodestructeur constituent, en psychopathologie, deux destins fréquents pour ce qui a été vécu sans pouvoir être digéré, métabolisé, élaboré par l'activité psychique. (Cf. Roussillon 1999)

On peut aussi s'interroger sur le caractère ' mélancoliforme , de l'impossible accès au maternel chez Barbara, qu'elle évoque brièvement dans son autobiographie :

Dans ma vie de femme j’ai échoué. Dans ma vie de mère j’ai échoué. J’ai longtemps senti dans mon ventre un vide glacé, j’ai longtemps jalousé les femmes enceintes et détesté les nouveau-nés. J'ai souvent marché la main posée sur mon ventre. Aujourd'hui, je pense que c'était sans doute le prix à payer et que ma vie a été malgré tout belle et intense. (Barbara 1998, 171s)

En psychanalyse, la mélancolie est définie comme une forme de deuil qui échoue : la haine inconsciente adressée à l'objet perdu, qui ne peut être assumée, est retournée contre le sujet lui-même, qui devient son propre objet de haine. (Cf. Freud 1968, 145s) Ce processus se traduit, en toute logique, par un vécu d'intense culpabilité, et donne lieu à des manœuvres 
d'auto-destructivité qui vont de l'auto-sabotage et des conduites à risque, aux passages à l'acte suicidaires dans les cas les plus graves. Dans cet extrait de son autobiographie, l'expression de " prix à payer » témoigne du poids de cette culpabilité sous-jacente chez Barbara : la privation de la maternité apparait comme une punition qu'il lui a fallu s'infliger à elle-même.

Enfin, il n'est pas possible d'évoquer le féminin mélancolique chez Barbara sans souligner l'omniprésence de la perte amoureuse, l'immense fragilité de son lien aux hommes. Barbara raconte et chante d'innombrables passions éphémères, si bien que Joël July considère la passion amoureuse comme la "thématique obsédante " (July 2004, 289) de son œuvre; en revanche, elle évoque peu de relations durables :

J'ai aimé la rencontre avec les hommes de ma vie [sic], la dualité, la complicité, le rire, la quiétude, l'impérieux besoin de reconquérir chaque matin, de rêver une vie à deux tout en sachant parfaitement que rien ni personne ne résisterait à mon piano, à mes thêâtres, à la route partagée avec d'autres. [...] Cet état d'amoureuse, je l'ai presque toujours connu. J'en avais besoin pour chanter. (Barbara 1998, 171)

Que-ce qui s'est joué pour elle de son économie psychique dans cet impossible accès à un investissement objectal qui soit autre chose que du registre de la passion? On pressent chez elle un rapport à la passion amoureuse à la fois antidépressif, addictif, et mortifere en ceci qu'elle agit, paradoxalement, à rebours du lien puisqu'elle semble contenir en elle-même sa propre fin, l'irrémédiable rupture. On rappellera ici l'origine étymologique du mot 'passion ', qui fait converger passivité et souffrance (du latin < patior `).

Cette position féminine mélancolique est figurée de façon exemplaire dans la comédie musicale Lily Passion jouée en 1986 avec Gérard Depardieu. Elle en résume l'histoire dans son autobiographie :

Lily est une chanteuse célèbre qui sillonne le monde; dans chaque ville où elle donne son tour de chant, un crime est commis. [...] Lily, hantée par ces assassinats, décide d'arrêter de chanter pour épargner des vies humaines. Mais la foule la réclame... (Barbara 1998, 179s)

Le plaisir du chant pour Barbara, hautement sexuel comme on l'a rappelé, est associé ici à la mort, la destructivité, la culpabilité, si bien qu'il doit y avoir renoncement (« extinction des mouvements pulsionnels » dans le féminin mélancolique, disait-on plus haut avec Catherine Chabert). Il s'agit d'une histoire d'amour avec un criminel, qui fait porter à l'amoureuse le poids de sa culpabilité : "Tu m’as rendue coupable de tes crimes abominables », chante-t-elle dans "Emmène-moi " (1986), nous renvoyant à nouveau à Ferenczi. On ne saurait mieux dire la confusion actif/passif, séducteur/séduit, et en dernier ressort victime/agresseur, qui hante si fréquemment la vie psychique des victimes d'inceste.

Dans « Le mal de vivre » (1965), Barbara utilise à nouveau les verbes « venir > et ‘ arriver ’, cette fois pour décrire la survenue de l'état mélancolique. À entendre ce texte à travers le 
prisme du féminin mélancolique, il est troublant de constater que Barbara y décrit l'envahissement dépressif avec les mêmes termes que l'abandon sexuel à un homme :

Ça ne prévient pas, ça arrive, ça vient de loin

Ça s'est trâné de rive en rive, la gueule en coin

Et puis un matin, au réveil, c'est presque rien

Mais c'est là, ça vous ensommeille, au creux des reins.

La douleur morale y est personnifiée, et Barbara n'a d'autre choix que de s'y abandonner, comme en d'autres temps à un homme.

\section{Conclusion : Barbara, femme coûte que coûte}

Barbara évoque sa capacité de dépassement du traumatisme (de résilience ?) suite à l'inceste paternel subi dans l'enfance. Par-delà la désintrication pulsionnelle caractéristique du trauma (et de ses résonnances mélancoliques que nous venons d'évoquer), Barbara témoigne de son féminin, envers et contre tout :

De ces humiliations infligées à l'enfance, de ces hautes turbulences, de ces descentes au fond du fond, j'ai toujours resurgi. Sûr, il m’a fallu un sacré goût de vivre, une sacrée envie d'être heureuse, une sacrée volonté d'atteindre le plaisir dans les bras d'un homme, pour me sentir un jour purifiée de tout, longtemps après. J'écris cela avec des larmes qui me viennent. $(1998,31 s)$

Certains de ses textes illustrent cette position féminine ` génitalisée ` à laquelle elle a accédé coûte que coûte, passionnément, faisant preuve d'une grande capacité de dépassement du trauma pour trouver la voie de la passivité et ‘ s'abandonner > à l'objet : c'est ce que suggère de façon emblématique l'un de ses grands succès, "Vienne » (1972a). Le texte est construit comme une série de lettres adressées à son compagnon laissé à Paris à la suite de quelque conflit conjugal (" notre ciel devenait si lourd »). Par le recours à la forme épistolaire, Barbara situe de fait l'objet dans une position de présence-absence : présent en tant que destinataire explicite du texte, qui lui est adressé précisément parce qu'il n'est pas là. Tout l'enjeu du message qu'elle adresse à cet absent s'organise autour de son abandon ou non à l'objet, le ressort de la tension dramatique se situant dans le basculement progressif : de « Non, je ne veux pas que tu viennes » à «Mon amour, il faut que tu viennes! ». Et l'on sent toute la jubilation qui est la sienne dans ce jeu consistant alternativement à tenir à distance et à ramener à soi l'objet d'amour (et son pendant : la tension suscitée chez l'amant, tantôt barré dans son désir, tantôt invité à y laisser libre cours). Difficile pour tout clinicien familier de la psychanalyse de ne pas penser, à l'écoute de ce balancement entre allers et venues, au célèbre « jeu de la bobine » décrit par Freud à l'orée du tournant des années 1920 (1920a). Freud observe 
son petit-fils de 18 mois jouer à lancer dans son lit une bobine de fil en criant " $\mathrm{O}$ ", qu'il ramène inlassablement à lui en prononçant le son " $\mathrm{A}$ » : Freud y entend respectivement les mots allemands 〈 fort > ( ( loin 〉) et < da > ( ( ici 〉). Ce jeu d'enfant, Freud en a fait l'emblème de la façon dont tout être humain s'efforce de maitriser le traumatisme de la perte de l'objet d'amour, en la répétant compulsivement. Cette appropriation de l'absence est ce qui permet progressivement à l'enfant humain de s'inscrire dans une pensée symbolique : les images, les fantasmes, les rêveries, les mots, les concepts, les pensées, autrement dit, tout ce qui fait la vie psychique, viennent représenter l'objet d'amour (en premier lieu, la mère), en lieu et place de sa présence réelle pour supporter son absence et son corrélat : la terreur de l'abandon (Freud parle en 1926 de ( Hilflosigkeit ), traduit en français par > état de détresse `, de < désaide > ; Freud 2009, 52).

Or chez Freud, l'observation de cette répétition, banale chez son petit-fils, a aussi servi de socle pour penser la clinique du traumatisme, qu'il rencontrait alors chez les névrosés de guerre de retour du front (on est en 1920) : Freud fait de cette compulsion à la répétition l'essence de la pulsion de mort, qui se traduit chez les soldats par la répétition des scènes de guerre, qui resurgissent telles quelles, nuit et jour, dans des cauchemars et des flashbacks. Or ce que nous donne à voir la poésie du féminin chez Barbara, c'est peut-être l'une des facettes de l'articulation des pulsions de vie et de mort, des forces de liaison et de déliaison, de la désintrication dont elles sont l'objet dans le trauma (libérant la pulsion de mort de son frein naturel, ainsi qu'en témoignent les diverses modalités de destructivité et d'autopunition qui caractérisent le féminin mélancolique), mais aussi de leur possible ré-intrication à la faveur du féminin génitalisé. Le plaisir hautement féminin de tenir l'autre à distance, de le faire languir pour mieux le laisser revenir, lui résister pour mieux finalement lui céder, le retenir avant de s'y abandonner, le perdre pour mieux le retrouver, ne relève-il pas d'une telle convergence (réussie) de la présence et de l'absence, de l'amour et de la perte, de la sexualité et de la mort ?

\section{Notes}

1 Pour les textes des chansons de Barbara, cf. July 2012. Les italiques dans les citations des chansons sont miens.

2 Du nom du père de Barbara.

3 C'est Ferenczi qui souligne.

\section{Bibliographie}

André, Jacques : Aux origines féminines de la sexualité. Paris : P.U.F., 2007.

Barbara : Il était un piano noir... Mémoires interrompus. Paris : Fayard, 1998.

Chabert, Catherine : Féminin mélancolique. Paris : P.U.F., 2007. 
Ferenczi, Sándor : "Confusion de langue entre les adultes et l'enfant ». In : Ferenczi, Sándor : Euvres complètes 1927-32. Vol. IV : Psychanalyse. Paris : Payot, 1996, 125-135.

Freud, Sigmund : "Deuil et mélancolie ». In : Freud, Sigmund : Métapsychologie. Paris : P.U.F., 1968, 145-171.

Freud, Sigmund : " Extrait de l'histoire d'une névrose infantile (L'homme aux loups) ». In : Freud, Sigmund : Cinq psychanalyses. Paris : P.U.F., 2001, 325-422.

Freud, Sigmund : "Au-delà du principe de plaisir». In : Freud, Sigmund : Essais de psychanalyse. Paris : Payot, 1973, 7-82.

Freud, Sigmund : "Sur la psychogenèse d'un cas d'homosexualité féminine ». In : Freud, Sigmund : Névrose, psychose et perversion. Paris : P.U.F., 1974, 245-270.

Freud, Sigmund : "Psychologie collective et analyse du moi ». In : Freud, Sigmund : Essais de psychanalyse. Paris : Payot, 1973, 83-175.

Freud, Sigmund : "Quelques conséquences psychiques de la différence anatomique entre les sexes ». In : Freud, Sigmund : La vie sexuelle. Paris : P.U.F., 2004, 123-132.

Freud, Sigmund : Inhibition, symptôme et angoisse. Paris : P.U.F., 2009.

Grimbert, Philippe : Psychanalyse de la chanson. Paris : Les belles lettres, 1996.

July, Joël : Les mots de Barbara. Aix-en-Provence : P.U.P., 2004.

July, Joël (éd.) : Barbara : l'intégrale. Paris : L'Archipel, 2012.

Korff-Sausse, Simone : "Des Gender Studies aux Disability Studies: repenser les catégories ». In : L'Esprit du temps 58,2 (2010), 37-52.

Poupart, Florent : «Roots and vicissitudes of psychic bisexuality ». In: International Journal of Psychoanalysis 97 (2016), 709-736.

Poupart, Florent : Erotisme et folie au féminin. Toulouse : P.U.M., 2017.

Roussillon, René : Agonie, clivage et symbolisation. Paris : P.U.F., 1999.

\section{Discographie}

Barbara : Dis, quand reviendras-tu ? CBS 62-660, 1964a (33 tours).

Barbara : Barbara chante Barbara. Philipps 77-806, 1964b (33 tours).

Barbara : Barbara. Philips 77859, 1965 (33 tours).

Barbara : Barbara. Philips 70-441, 1967 (33 tours).

Barbara : L’Aigle Noir. Philips 6311-084, 1970 (33 tours).

Barbara : La Fleur d'amour. Philips 6325-004, 1972a (33 tours).

Barbara : Amours incestueuses. Philips 6332-119, 1972b (33 tours).

Barbara : Seule. Philips 6313-134, 1981 (33 tours).

Barbara : Lily Passion. Philips 826-825, 1986 (2 CD). 